\title{
Improving the Production of 9,21-dihydroxy-20- methyl-pregna-4-en-3-one from Phytosterols in Mycobacterium Neoaurum by Modifying Multiple Genes and Improving the Intracellular Environment
}

\section{Chen-Yang Yuan}

SARI: Chinese Academy of Sciences Shanghai Advanced Research Institute

Zhi-Guo Ma

SARI: Chinese Academy of Sciences Shanghai Advanced Research Institute Jing-Xian Zhang

SARI: Chinese Academy of Sciences Shanghai Advanced Research Institute

\section{Xiang-Cen Liu}

SARI: Chinese Academy of Sciences Shanghai Advanced Research Institute

\section{Gui-Lin Du}

SARI: Chinese Academy of Sciences Shanghai Advanced Research Institute Jun-Song Sun

SARI: Chinese Academy of Sciences Shanghai Advanced Research Institute https://orcid.org/00000002-7339-3250

\section{Ji-Ping Shi}

SARI: Chinese Academy of Sciences Shanghai Advanced Research Institute

\section{Bao-Guo Zhang ( $\square$ zhangbg@sari.ac.cn )}

Shanghai Advanced Research Institute https://orcid.org/0000-0002-0800-0342

\section{Research}

Keywords: 9,21-dihydroxy-20-methyl-pregna-4-en-3-one (9-OH-4-HP), kstd, hsd4A, fadA5, intracellular environment

Posted Date: August 10th, 2021

DOl: https://doi.org/10.21203/rs.3.rs-783513/v1

License: (c) (i) This work is licensed under a Creative Commons Attribution 4.0 International License. Read Full License 


\section{Abstract}

\section{Background}

Steroid drugs are particularly important for disease prevention and clinical treatment. However, traditional chemical methods are rarely implemented during the whole synthetic process to generate steroid intermediates due to the intricate steroid structure. Novel steroid drug precursors and their ideal bacterial strains for industrial production have yet to be developed. Among these, $9-\mathrm{OH}-4-\mathrm{HP}$ is a potential steroid drug precursor for the synthesis of corticosteroids. In this study, a combined strategy of blocking $\Delta^{1}$ dehydrogenation and the $\mathrm{C} 19$ pathway as well as improving the intracellular environment was investigated to construct an effective 9-OH-4-HP-producing strain.

\section{Results}

A $\Delta^{1}$-dehydrogenation-deficient strain of wild-type Mycobacterium neoaurum DSM 44074 produces 9-0H4-HP with a molar yield of $4.8 \%$. hsd $4 A$, encoding a $\beta$-hydroxyacyl-CoA dehydrogenase, and fadA5 encoding an acyl-COA thiolase, were separately knocked out to block the C19 pathway in the $\Delta^{1}$ dehydrogenation-deficient strain. The two engineered strains could accumulate $0.59 \mathrm{~g} \mathrm{~L}^{-1}$ and $0.47 \mathrm{~g} \mathrm{~L}^{-1}$ 9-OH-4-HP from $1 \mathrm{~g} \mathrm{~L}^{-1}$ phytosterols. Furthermore, hsd $4 A$ and fadA5 were knocked out simultaneously in the $\Delta^{1}$-dehydrogenation-deficient strain. The 9-OH-4-HP production from the Hsd4A and FadA5 doubledeficient strain was $11.9 \%$ higher than that of the Hsd4A -deficient strain and $40.4 \%$ higher than that of the strain with FadA5 deficiency, and its selectivity reached $94.9 \%$. Subsequently, the catalase katE from Mycobacterium and an NADH oxidase, nox, from Bacillus subtilis were overexpressed to improve the intracellular environment. Ultimately, 9-OH-4-HP production reached $3.58 \mathrm{~g} \mathrm{~L}^{-1}$ from $5 \mathrm{~g} \mathrm{~L}^{-1}$ phytosterols, and the selectivity of 9-OH-4-HP improved to $97 \%$.

\section{Conclusion}

hsd $4 A$ and fadA5 are key enzymes in the $C 19$ pathway for phytosterol side chain degradation. Deletion of hsd $4 A$ and fadA5 could almost entirely block the C19 pathway. Improving the intracellular environment of Mycobacterium during phytosterol bioconversion could accelerate the conversion process and enhance the productivity of target sterol derivatives.

\section{Background}

Steroid drugs, including mineralocorticoids, glucocorticoids, and sex hormones, are crucial in the prevention and clinical treatment of various diseases, including diabetes, asthma, neurodegenerative disorders, cancer, hormone metabolic syndrome, and many others [1,2]. Steroid drugs represent one of the best-selling classes of drugs and are the second-largest pharmaceutical on the market. Global pharmaceutical industry predictions indicate that many drugs based on steroid compounds will retain market leadership in the future. 
In industrial manufacturing, two major valuable intermediates of sterols, C19 steroids and C22 steroids, can be used to synthesize sex and adrenocortical hormones. However, traditional chemical methods are rarely implemented in the whole synthetic processes of modifying steroid intermediates due to the intricate steroid structure. Thus, the pursuit of novel steroid drug precursors has intrigued researchers. Certain C22 steroids, including 21-hydroxy-20-methyl-pregna-4-en-3-one (4-HP), 21-hydroxy-20-methylpregna-1,4-dien-3-one (1,4-HP), and 9,21-dihydroxy-20-methyl-pregna-4-en-3-one (9-OH-4-HP), are ideal precursors for steroid drug synthesis [3]. Among these steroids, 9-OH-4-HP is a highly valuable steroid derivative for the synthesis of corticosteroids because of its substituents at positions 9 and 21. Early studies identified 9-OH-4-HP as a by-product of sterol bioconversion to generate 9-hydroxy steroid derivatives in several Mycobacterium species, such as Mycobacterium sp. 2-4M, which produces a 1.5\%-1.6\% molar yield of 9-OH-4-HP [4]. Mycobacterium sp. VKM Ac-1815D, Mycobacterium sp. VKM Ac1817D, and Mycobacterium fortuitum ATCC-6842 have also been reported to accumulate a small amount of 9-OH-4-HP during the 9-OH-AD production process [4-6]. However, ideal industrial strains have yet to be developed.

Actinomycetes, such as Mycobacterium, share similar sterol metabolic pathways. The initial step of sterol metabolism is the conversion of sterols to 4-en-3-sterone, followed by degradation of the sterol side chain and cleavage of the steroid skeleton[3, 7,8$]$. The cleavage of the steroid $B$ ring is performed by $\Delta^{1}$ dehydrogenation catalysed by 3-ketosteroid-1(2)-dehydrogenase (KstD) and C9-hydroxylation catalysed by 9 a-hydroxylase $(\mathrm{KSH})[9,10]$. Consequently, blocking $\mathrm{C} 9$ hydroxylation resulted in various $\mathrm{C} 19$ steroids. 9a-Hydroxy derivatives, such as 9-hydroxy-androst-4-ene-3,17-dione (9-OH-AD), are important precursors in the manufacture of several modern glucocorticoid drugs bearing a halogen at the 9 a position [11]. An engineered strain of Mycobacterium. neoaurum ATCC 25795 in which kstDs are knocked out can accumulate $6.02 \mathrm{~g} \mathrm{~L}^{-1}$ 9-OH-AD as the main product from $15 \mathrm{~g} \mathrm{~L}^{-1}$ phytosterols [12]. kstd 1 and $k s t d 2$ have also been reported to be effective for 9-OH-AD degradation in Rhodococcus rhodochrous DSM43269 [13]. By overexpression of certain related genes and knockout of $k s t D s$, the production of 9$\mathrm{OH}-\mathrm{AD}$ increased by $45 \%$ in M. neoaurum MS136 [14]. A few wild-type strains of Mycobacterium have been reported to be able to produce 9-OH-AD from plant or animal sterols via a single-step microbial conversion [4]. Normally, 9a-hydroxy derivatives can be obtained from industrial strains in which kstDs are deleted.

In contrast, side chain degradation is rather complicated. Some key enzymes involved in the side chain metabolic pathway remain uncertain, limiting the comprehensive understanding of the process. Dual competing pathways, the overwhelming C19 steroid pathway and the C22 steroid pathway, are involved in phytosterol side chain degradation. Recently, the 17-hydroxysteroid/22-OH-BNC-CoA dehydrogenase Hsd4A was found to be relevant during C22 steroid formation [3]. Inactivation of Hsd4A enabled the production of $\mathrm{C} 22$ steroids from sterols. For example, $M$. neoaurum NWIB-XII accumulates androst-4-ene3,17-dione (AD) and androst-1,4-diene-3,17-dione (ADD), which are both $\mathrm{C19}$ steroids, as the main products after culture with cholesterol. The $h s d 4 A$ knockout strain accumulates 4-HP and 1,4-HP as the main products. Nevertheless, $\mathrm{C} 19$ steroids still accumulated in the $h s d 4 A$ knockout strain from 
phytosterols, indicating incomplete blockage of the C19 steroid pathway. An Hsd4A- and KstDs-deficient strain of M. neoaurum ATCC 25795 gives a $32 \%$ molar yield of $9-\mathrm{OH}-4-\mathrm{HP}$ and a 15\% molar yield of $9-\mathrm{OH}-$ $A D$ from $40 \mathrm{~g} \mathrm{~L}^{-1}$ phytosterols [3]. FadA5, a thiolase, was reported to be essential for the production of AD/ADD from cholesterol by $M$. tuberculosis H37Rv [15]. A FadA5-deficient strain of M. neoaurum NwIBXII also accumulates 4-HP and 1,4-HP as the main products. Thus, the deletion of fadA5 may contribute to further blockage of the $C 19$ pathway. Therefore, hsd $4 A$ and fadA5 are important targets for modification by genetic engineering to develop microorganisms that can transform sterols into the valuable steroidal intermediate 9-OH-4-HP.

On the other hand, phytosterols and their metabolites are toxic to cells, as they could inhibit cell growth and biocatalytic activity [16]. A steady-state intracellular environment could be beneficial for phytosterol degradation by Mycobacterium. Toxic steroid intermediates cause cells to produce reactive oxygen species (ROS), including hydrogen peroxide $\left(\mathrm{H}_{2} \mathrm{O}_{2}\right)$, during aerobic metabolism. A high level of $\mathrm{H}_{2} \mathrm{O}_{2}$ might harm cell growth, hence slowing the rate of phytosterol degradation and decreasing the yield of metabolites [17], and vice versa. For example, elimination of $\mathrm{H}_{2} \mathrm{O}_{2}$ from $M$. neoaurum JC-12 increased the 4 -HP yield by $24 \%$. In addition, during the phytosterol degradation process, intracellular nicotinamide adenine dinucleotides (NAD ${ }^{+}$and $\left.N A D H\right)$ are consumed, which participate in multistep reactions during phytosterol degradation, such as when dehydrogenation occurs. NAD ${ }^{+} / \mathrm{NADH}$ regeneration and maintenance of the redox balance are considered the rate-limiting factors in the steroid degradation pathway $[17,18]$. Manipulation of $\mathrm{NAD}^{+} / \mathrm{NADH}$ contents could enhance the production of $A D$ and $A D D$ to various degrees [17-19]. Overexpression of NADH oxidase in M. neoaurum JC-12 increased ADD production by $43 \%$ [17], and an increase in the amounts of the ratio of $\mathrm{NAD}^{+} / \mathrm{NADH}$ in $M$. neoaurum TCCC 11978 enhanced the productivity of $A D D$ by $93 \%$. Thus, the elimination of $\mathrm{H}_{2} \mathrm{O}_{2}$ and regeneration of $N A D^{+}$ could contribute to higher concentrations of phytosterol metabolites.

Herein, an engineered strain of $M$. neoaurum DSM 44074, which is a sterol consumer, was constructed for the bioconversion of phytosterols to 9-OH-4-HP. A kstDs knockout strain was constructed based on $M$. neoaurum DSM 44074, and the C19 steroid pathway was further blocked by knocking out both $h s d 4 A$ and fadA5. By improving the intracellular environment, an efficient 9-OH-4-HP-producing strain was generated. This strain may contribute to the development of steroid drug precursors.

\section{Results}

\section{Accumulation of 9a-hydroxy derivatives}

To eliminate $\Delta^{1}$-dehydrogenation and accumulate 9 a-hydroxy derivatives from phytosterols, $k s t D s$ were identified and knocked out from the genome of the wild-type strain M. neoaurum DSM 44074, a steroiddegrading Mycobacterium that can completely degrade phytosterols to produce $\mathrm{CO}_{2}$ and $\mathrm{H}_{2} \mathrm{O}$ [7]. The genome of $M$. neoaurum DSM 44074 was sequenced as described in the Methods section. Three putative kstD genes (gene 5102 for $k s t d 1$, gene 5236 for kstd2, and gene 5233 for kstd3) were identified in 
M. neoaurum DSM 44704. kstDs was successfully knocked out from the genome of $M$. neoaurum DSM 44704 as described in the Methods section, and the mutant strain $\Delta K s t D$ was obtained. The cell growth of the $\Delta k s t D$ strain showed no significant difference from that of the wild-type strain (Fig. S1). The wildtype $M$. neoaurum DSM 44074 strain and the genetically modified strain $\Delta k s t D$ were incubated with phytosterols for $168 \mathrm{~h}$. The resulting metabolites were extracted from the culture supernatants and analysed by HPLC. Compared with the wild-type strain M. neoaurum DSM 44074, which showed no detectable product by HPLC analysis (Fig. 2a), the $\triangle k s t D$ strain produced 9-OH-AD as the main product with a retention time of 4.2 min (Fig. 2a, peak A), along with 9-OH-4-HP as a by-product with a retention time of $7.1 \mathrm{~min}$ (Fig. 2a, peak B). No ADD was detected during phytosterol bioconversion by $\Delta k s t D$, which indicated that the phytosterol degradation pathway was interrupted because of the elimination of $\Delta^{1}$ dehydrogenation by $k s t D s$ knockout. When MP01 medium plus $1 \mathrm{~g} \mathrm{~L}^{-1}$ phytosterols was used for incubation of the $\Delta k s t D$ strain, $0.62 \mathrm{~g} \mathrm{~L}^{-1} 9-\mathrm{OH}-\mathrm{AD}$ and $0.04 \mathrm{~g} \mathrm{~L}^{-1} 9-\mathrm{OH}-4-\mathrm{HP}$ were produced within $60 \mathrm{~h}$ (Fig. 3a and $3 \mathrm{~b}$ ). The molar yield of 9-OH-AD reached $84.9 \%$. 9-OH-4-HP was the major by-product duringphytosterol bioconversion by $\Delta k s t D$, but the molar yield of $9-\mathrm{OH}-4-\mathrm{HP}$ was only $4.8 \%$, and the selectivity of 9-OH-4-HP was 5.6\% (Table 2). Thus, 9a -hydroxy derivatives successfully accumulated during phytosterol bioconversion by Mycobacterium, but the purity and yield of 9-OH-4-HP remained unsatisfactory.

\section{Construction of a 9-OH-4-HP-producing strain}

Dual pathways, the $\mathrm{C} 19$ steroid pathway and the $\mathrm{C} 22$ steroid pathway, competes during phytosterol side chain degradation. The $\mathrm{C} 19$ steroid pathway is the dominant pathway in M. neoaurum DSM 44074, which could be confirmed that $\Delta k s t D$ produces $9-\mathrm{OH}-\mathrm{AD}$ as the main product along with 9-OH-4-HP as a by-product after culture with phytosterols. The two pathways diverge at 22hydroxy-3,24-dioxo-4-ene-cholest-COA (22-OH-24-CDOE-COA), which could be $\Delta^{22}$-dehydrogenated by the $\beta$-hydroxyacyl-CoA dehydrogenase Hsd4A to generate 3,22,24-trioxo-4-ene-cholest-COA (24-CTOE-COA). 24-CTOE-COA could subsequently be catalysed by the thiolase FadA5, leading the phytosterol degradation pathway to the C19 pathway.

Thus, to construct a 9-OH-4-HP-producing strain, $h s d 4 A$ and fadA5 were identified in the genome of $M$. neoaurum DSM 44074 and separately knocked out in $\triangle k s t D$, resulting in the strains $\triangle k s t D \triangle h s d 4 A$ and $\triangle k s t D \triangle$ fadA5.

The cell growth of $\Delta k s t D \Delta h s d 4 A$ and $\triangle k s t D \Delta$ fadA5 showed no significant difference from that of the wild-type strain $M$. neoaurum DSM 44074 (Fig. S1). The strains $\Delta k s t D \Delta h s d 4 A$ and $\Delta k s t D \Delta f a d A 5$ were cultured with phytosterols for $168 \mathrm{~h}$, and the metabolites were analysed by HPLC (Fig. 2b). As shown in Fig. 3b, 9-OH-4-HP successfully accumulated in both strains $\Delta k s t D \Delta h s d 4 A$ and $\Delta k s t D \Delta f a d A 5$. The selectivity of $9-\mathrm{OH}-4-\mathrm{HP}$ from the $\Delta k s t D \Delta h s d 4 A$ and $\Delta k s t D \Delta$ fad $A 5$ strains were $88.6 \%$ and $86.0 \%$, respectively. Nevertheless, both strains still showed only a small amount of 9-OH-AD accumulation (Fig. 3a). The selectivity of $9-\mathrm{OH}-\mathrm{AD}$ from strains $\Delta k s t D \Delta h s d 4 A$ and $\Delta k s t D \Delta$ fadA5 were $7.2 \%$ and $8.0 \%$, 
respectively. After culture with $1 \mathrm{~g} \mathrm{~L}^{-1}$ phytosterols in MP01 medium, the strain $\Delta k s t D \Delta h s d 4 A$ accumulated $0.59 \mathrm{~g} \mathrm{~L}^{-1}$ 9-OH-4-HP and $0.13 \mathrm{~g} \mathrm{~L}^{-1}$ 9-OH-AD, while $0.47 \mathrm{~g} \mathrm{~L}^{-1}$ 9-OH-4-HP and $0.08 \mathrm{~g} \mathrm{~L}^{-1}$ 9-OH$\mathrm{AD}$ were obtained from strain $\triangle k s t D \triangle$ fadA5. The molar yields of 9-OH-4-HP and 9-OH-AD from strain $\Delta k s t D \triangle$ fadA5 were both lower than those from strain $\Delta k s t D \Delta h s d 4 A$. The molar yield of 9-0H-4-HP from strain $\triangle k s t D \Delta$ fadA5 was $20.3 \%$ lower than that from strain $\Delta k s t D \Delta h s d 4 A$, and the molar yield of $9-\mathrm{OH}-\mathrm{AD}$ from strain $\Delta k s t D \triangle f a d A 5$ which was $38.5 \%$ lower than that from strain $\Delta k s t D \Delta h s d 4 A$.

Considering that 9-OH-AD still accumulated in both the $\Delta k s t D \Delta h s d 4 A$ and $\triangle k s t D \Delta$ fadA5 strains, the C19 steroid pathway of the phytosterol degradation pathway was not completely blocked in either strain. Thus, to enhance the purity and productivity of 9-OH-4-HP and obstruct the yield of 9-OH-AD, hsd4A and fadA5 were both knocked out in strain $\triangle k s t D$, and strain $\triangle k s t D \Delta h s d 4 A \triangle \mathrm{fad} A 5$ was obtained. The cell growth of the $\Delta k s t D \Delta h s d 4 A \Delta f a d A 5$ strain showed a trend similar to that of the wild-type strain M. neoaurum DSM 44074 (Fig. S1). As shown in Fig. 2b, after culture with phytosterols and metabolites being analysed by HPLC, the strain $\triangle k s t D \Delta h s d 4 A \triangle$ fad $A 5$ accumulated 9-OH-4-HP as the main product, while the accumulation of 9-OH-AD was significantly decreased compared with the strains $\triangle k s t D \Delta h s d 4 A$ and $\Delta k s t D \Delta$ fadA5. The selectivity of 9-OH-4-HP and 9-OH-AD from strain $\Delta k s t D \Delta h s d 4 A \Delta$ fadA5 were $94.9 \%$ and $2.0 \%$, respectively. The purity of $9-0 \mathrm{H}-4$-HP from strain $\Delta k s t D \Delta h s d 4 A \Delta$ fad $A 5$ was higher than those from strains $\Delta k s t d \Delta h s d 4 A$ and $\Delta k s t D \Delta$ fadA5. After culture with $1 \mathrm{~g} \mathrm{~L}^{-1}$ phytosterols, $0.66 \mathrm{~g} \mathrm{~L}^{-1}$ 9-OH-4-HP was obtained from strain $\triangle k s t D \Delta h s d 4 A \triangle$ fadA5, which is $11.9 \%$ more than that from strain $\Delta k s t D \Delta h s d 4 A$ and $40.4 \%$ more than that from strain $\triangle k s t D \Delta$ fadA5 (Fig. 3b). The selectivity and production of 9-OH-4-HP from strain $\triangle k s t D \Delta h s d 4 A \triangle$ fadA5 were both higher than those from strains $\triangle k s t D \Delta h s d 4 A$ and $\triangle k s t D \triangle f a d A 5$, indicating that double knockout of $h s d 4 A$ and fadA5 could effectively block the accumulation of $A D$ homologues.

Moreover, to verify the functions of $h s d 4 A$ and fadA5 during phytosterol degradation, $\triangle k s t D \triangle h s d 4 A$ $h s d 4 A$, the $h s d 4 A$ complementation strain, and $\triangle k s t D \triangle f a d A 5-f a d A 5$, the fadA5 complementation strain, were also constructed. As shown in Figure 2c, when the two complementation strains were cultured with phytosterols and metabolites being analysed by HPLC, the accumulation of 9-OH-AD was recovered. The purities of 9-OH-AD from $\Delta k s t D \Delta h s d 4 A$ - $h s d 4 A$ and $\Delta k s t D \Delta$ fadA5-fad $A 5$ were $90.0 \%$ and $88.5 \%$, respectively, which is nearly consistent with those of strain $\Delta k s t D$. These results indicated that Hsd4A and FadA5 were key enzymes in the C19 steroid pathway during phytosterol degradation. Phylogenetic trees of Hsd4A and FadA5 were constructed to elucidate evolutionary relationship of the two enzymes (Fig. S2).

\section{Evaluation of the 9-0H-4-HP producer}

After culture with $1 \mathrm{~g} \mathrm{~L}^{-1}$ phytosterols, the molar yield of 9-OH-4-HP from $\Delta k s t D \Delta h s d 4 A \Delta$ fadA 5 was $78.9 \%$. To evaluate the ability of $\triangle k s t D \Delta h s d 4 A \Delta$ fadA5 to transform phytosterols into 9-OH-4-HP, different concentrations of phytosterols were incubated with $\Delta k s t D \Delta h s d 4 A \triangle \mathrm{fad} A 5$. 
As shown in Fig. 4b, the yields of 9-OH-4-HP from the bioconversion of $2 \mathrm{~g} \mathrm{~L}^{-1}, 5 \mathrm{~g} \mathrm{~L}^{-1}, 8 \mathrm{~g} \mathrm{~L}^{-1}$, and $10 \mathrm{~g} \mathrm{~L}^{-1}$ phytosterols by $\Delta k s t D \Delta h s d 4 A \Delta$ fadA5 were $1.43 \mathrm{~g} \mathrm{~L}^{-1}, 2.78 \mathrm{~g} \mathrm{~L}^{-1}, 1.98 \mathrm{~g} \mathrm{~L}^{-1}$, and $1.73 \mathrm{~g} \mathrm{~L}^{-1}$, respectively. 9$\mathrm{OH}-\mathrm{AD}$ was also obtained during the incubation, showing yields of $0.06 \mathrm{~g} \mathrm{~L}^{-1}, 0.10 \mathrm{~g} \mathrm{~L}^{-1}, 0.03 \mathrm{~g} \mathrm{~L}^{-1}$, and $0.04 \mathrm{~g} \mathrm{~L}^{-1}$, respectively (Fig. 4a). The productivity of 9-OH-4-HP was enhanced as the concentration of phytosterols increased up to $5 \mathrm{~g} \mathrm{~L}^{-1}$. However, at phytosterol concentrations of $8 \mathrm{~g} \mathrm{~L}^{-1}$ and $10 \mathrm{~g} \mathrm{~L}^{-1}$, the productivity of 9-OH-4-HP decreased, showing results only slightly higher than that with $2 \mathrm{~g} \mathrm{~L}^{-1}$ phytosterols, and was obviously lower than that with $5 \mathrm{~g} \mathrm{~L}^{-1}$ phytosterols. The molar yields of 9-OH-4-HP from different concentrations of phytosterols are listed in Table 3. A downward trend in the molar yield of 9-OH-4-HP appeared as the concentration of phytosterols increased from $2 \mathrm{~g} \mathrm{~L}^{-1}$ to $10 \mathrm{~g} \mathrm{~L}^{-1}$. However, the purity of 9-OH-4-HP remained stable. Previous research has reported that phytosterols and their metabolites could be noxious to cells during bioconversion [16, 20-22], which might account for the poor performance of $\Delta k s t D \Delta h s d 4 A \triangle$ fadA 5 during phytosterol bioconversion as the concentration of phytosterols increased.

\section{Intracellular environmental balance contributes to higher 9- $\mathrm{OH}-4-\mathrm{HP}$ production}

The 9-OH-4-HP-producing strain $\Delta k s t D \Delta h s d 4 A \Delta$ fadA5 did not perform well during the bioconversion of phytosterols when the concentration of phytosterols was higher than $2 \mathrm{~g} \mathrm{~L}^{-1}$. This might be due to multiple factors that influence the bioconversion of phytosterols.

A series of redox reactions occur during phytosterol degradation, which use oxygen as an electron acceptor, and cholesterol dehydrogenases/isomerases require intracellular nicotinamide adenine dinucleotides (NAD ${ }^{+}$and $\left.N A D H\right)$ as cofactors $[23,24]$. NAD ${ }^{+}$and NADH play important roles during phytosterol transformation. They act in many oxidation-reduction reactions and regulate various enzymatic activities and genetic processes. The intracellular $\mathrm{NAD}^{+}$concentration decreased due to its consumption. Therefore, $\mathrm{NAD}^{+}$and $\mathrm{NADH}$ have critical effects on the maintenance of the intracellular redox balance. Regeneration of $\mathrm{NAD}^{+}$and enhancement of the $\mathrm{NAD}{ }^{+} / \mathrm{NADH}$ ratio may be of great assistance during phytosterol transformation.

In addition, hydrogen peroxide ( $\mathrm{H} 2 \mathrm{O} 2)$ is produced due to incomplete oxidation during aerobic metabolism and the regeneration of flavin adenine dinucleotide (FAD) during the phytosterol transformation process [17]. A high level of $\mathrm{H} 2 \mathrm{O} 2$ can damage proteins, DNA, and lipids in cells, resulting in inhibition of cell growth and metabolite yield [25].

To enhance the ability of strain $\Delta k s t D \Delta h s d 4 A \Delta$ fadA5 to transform phytosterols into 9-OH-4-HP, the catalase katE from DSM 44074 and the NADH oxidase nox from Bacillus subtilis [17] were co-expressed in strain $\Delta k s t D \Delta h s d 4 A \Delta$ fadA5to construct strain $\Delta k s t D \Delta h s d 4 A \Delta$ fadA5-NK. 
The extracellular $\mathrm{H}_{2} \mathrm{O}_{2}$ concentrations of the two strains $\Delta k s t D \Delta h s d 4 A \Delta$ fadA5 and $\Delta k s t D \Delta h s d 4 A \Delta$ fadA5NK were measured when they were cultured with $5 \mathrm{~g} \mathrm{~L}^{-1}$ phytosterols for $168 \mathrm{~h}$. As shown in Figure $5 \mathrm{~b}$, the extracellular $\mathrm{H}_{2} \mathrm{O}_{2}$ concentration of strain $\Delta k s t D \Delta h s d 4 A \Delta$ fadA5 showed an upward trend during the bioconversion process. The extracellular $\mathrm{H}_{2} \mathrm{O}_{2}$ concentration increased from $0.59 \mu \mathrm{mol} \mathrm{L}{ }^{-1}$ at the beginning to $1.05 \mu \mathrm{mol} \mathrm{L}-1$ after $168 \mathrm{~h}$ and reached a peak of $1.10 \mu \mathrm{mol} \mathrm{L}^{-1}$ at $120 \mathrm{~h}$. In contrast, the extracellular $\mathrm{H}_{2} \mathrm{O}_{2}$ concentration of strain $\Delta k s t D \Delta h s d 4 A \Delta$ fadA5-NK remained nearly stable during the bioconversion process at approximately $0.51 \mu \mathrm{mol} \mathrm{\textrm {L } ^ { - 1 }}$. Therefore, the overexpression of $k a t E$ eliminated excessive extracellular $\mathrm{H}_{2} \mathrm{O}_{2}$. Moreover, to verify the toxicity of $\mathrm{H}_{2} \mathrm{O}_{2}$, cell growth of the strains $\triangle k s t D \Delta h s d 4 A \Delta f a d A 5$ and $\Delta k s t D \Delta h s d 4 A \triangle f a d A 5$-NK was also measured. As shown in Figure $5 \mathrm{a}$, the biomass of strain $\Delta k s t D \Delta h s d 4 A \Delta$ fadA5-NK was higher than that of strain $\Delta k s t D \Delta h s d 4 A \Delta f a d A 5$, indicating that the elimination of extracellular $\mathrm{H}_{2} \mathrm{O}_{2}$ could help with cell growth.

Likewise, the $N A D^{+} / N A D H$ ratios of the strains $\Delta k s t D \Delta h s d 4 A \Delta$ fadA and $\triangle k s t D \Delta h s d 4 A \Delta$ fadA-NK were also measured after they were cultured with $5 \mathrm{~g} \mathrm{~L}^{-1}$ phytosterols for $168 \mathrm{~h}$. As shown in Figure $5 \mathrm{c}$, the $\mathrm{NAD}^{+} / \mathrm{NADH}$ ratio of strain $\triangle k s t D \Delta h s d 4 A \triangle \mathrm{fadA-NK}$ was consistently higher than that of strain $\Delta k s t D \triangle h s d 4 A \triangle$ fadA by at least $10.9 \%$. At $96 \mathrm{~h}$, the NAD ${ }^{+} / \mathrm{NADH}$ ratio was enhanced by $25.4 \%$ in strain $\Delta k s t D \Delta h s d 4 A \Delta$ fadA-NK compared with strain $\Delta k s t D \Delta h s d 4 A \Delta f a d A$. The overexpression of nox could significantly influence the $\mathrm{NAD}^{+} / \mathrm{NADH}$ ratio during phytosterol bioconversion.

9-OH-4-HP productivity was also measured to test whether overexpression of katE and nox could enhance the ability of $\Delta k s t D \Delta h s d 4 A \Delta$ fadA to transform phytosterols into 9-OH-4-HP. The recombinant strain $\Delta k s t D \Delta h s d 4 A \Delta$ fadA-NK was cultured with $1 \mathrm{~g} \mathrm{~L}^{-1}, 2 \mathrm{~g} \mathrm{~L}^{-1}, 5 \mathrm{~g} \mathrm{~L}^{-1}, 8 \mathrm{~g} \mathrm{~L}^{-1}$, and $10 \mathrm{~g} \mathrm{~L}^{-1}$ phytosterols for 168 $\mathrm{h}$, and the productivity of 9-OH-4-HP was measured every $24 \mathrm{~h}$. As shown in Figure $5 \mathrm{~d}$, the final productivities of 9-OH-4-HP from $1 \mathrm{~g} \mathrm{~L}^{-1}, 2 \mathrm{~g} \mathrm{~L}^{-1}, 5 \mathrm{~g} \mathrm{~L}^{-1}, 8 \mathrm{~g} \mathrm{~L}^{-1}$, and $10 \mathrm{~g} \mathrm{~L}^{-1}$ phytosterols were $0.68 \mathrm{~g} \mathrm{~L}^{-1}$, $1.53 \mathrm{~g} \mathrm{~L}^{-1}, 3.58 \mathrm{~g} \mathrm{~L}^{-1}, 2.51 \mathrm{~g} \mathrm{~L}^{-1}$, and $2.73 \mathrm{~g} \mathrm{~L}^{-1}$, respectively. Compared with $\Delta k s t D \Delta h s d 4 A \triangle$ fadA cultured with the same concentrations of phytosterols, the productivities of $9-\mathrm{OH}-4-\mathrm{HP}$ were enhanced by $3.03 \%$, $6.99 \%, 28.7 \%, 26.8 \%$, and $57.8 \%$. The highest yield of $9-0 \mathrm{H}-4-\mathrm{HP}$ was obtained when strain $\Delta k s t D \Delta h s d 4 A \triangle$ fadA-NK was cultured with $5 \mathrm{~g} \mathrm{~L}^{-1}$ phytosterols, with a molar yield that reached $85.5 \%$, which was $28.8 \%$ higher than that of $\Delta k s t D \Delta h s d 4 A \Delta$ fadA. Moreover, no significant difference in the productivity of 9-OH-AD was observed between the two strains $\Delta k s t D \triangle h s d 4 A \triangle$ fadA5 and $\Delta k s t D \Delta h s d 4 A \triangle$ fadA5-NK (Fig. $5 \mathrm{e}$ ), indicating that the purity of 9-OH-4-HP was also enhanced during phytosterol bioconversion by the strain $\Delta k s t D \Delta h s d 4 A \Delta$ fadA5-NK. All of the results above confirm that regulation of the intracellular $\mathrm{NAD}^{+} / \mathrm{NADH}$ ratio and $\mathrm{H}_{2} \mathrm{O}_{2}$ levels could be an effective way to improve sterol transformation efficiency and the production of steroid intermediates.

\section{Discussion}

By genome sequencing, three $k s t D s$ were found in M. neoaurum DSM 44074. kstD2 and $k s t D 3$ in $M$. neoaurum DSM 44074 showed $100 \%$ similarity with those in M. neoaurum ATCC 25795, a strain that was 
deemed to be the same strain as M. neoaurum DSM 44074. However, kstD1 in M. neoaurum DSM 44074 showed 5 mismatches with that in $M$. neoaurum DSM 44074, causing 3 amino acid changes. When the kstds knockout strain of $M$. neoaurum DSM 44074 was cultured with phytosterols, AD and 4-HP were nearly undetectable in the final products. The $k s t d$ knockout strain accumulated $9-\mathrm{OH}-\mathrm{AD}$ as the main product and 9-OH-4-HP as a by-product. Certain other strains have also been reported to accumulate 9$\mathrm{OH}-\mathrm{AD}$ from phytosterols. Mycobacterium sp. 2-4M [26] showed a $50 \%$ molar yield of $9-\mathrm{OH}-\mathrm{AD}$, a $22 \%$ molar yield of $A D$ and a $2 \%$ molar yield of 4-HP from $5 \mathrm{~g} \mathrm{~L}^{-1}$ sitosterol [27]. In a kstds knockout strain of M. neoaurum ATCC 25795 , a $55 \%$ molar yield of $9-O H-A D$ and a $15 \%$ molar yield of AD were obtained from $15 \mathrm{~g} \mathrm{~L}^{-1}$ phytosterols. Compared with other $9-\mathrm{OH}-\mathrm{AD}$-producing strains, the purity and molar yield of 9-OH-AD from $\triangle k s t D$ after culture with phytosterols were notably higher. The accumulation of $A D$ from $k s t D s$ knockout strains might be due to residual $\Delta^{1}$-dehydrogenation activity. The genome of $R$. ruber contains at least two other possible ORFs other than $k s t D 1, k s t D 2$, and $k s t D 3$ with certain identity to $k s t D s$ (approximately 38\%) [28]. The existence of more than $3 \mathrm{KstDs}$ has also been reported for other Rhodococcus species, such as R. jostii Rha1 [29]. Thus, inactivation of all KstD activities ought to be the fundamental premise to develop promising 9 a-hydroxy derivatives.

Due to dual competing pathways, the dominant C19 steroid pathway and the C22 steroid pathway exist in the phytosterol degradation pathway. 9-OH-4-HP is usually produced as a by-product in 9-OH-ADproducing strains. M. V. Donova reported a wild-type strain Mycobacterium sp. 2-4M, which produces 9$\mathrm{OH}-\mathrm{AD}$ as the major product with a 1.5\%-1.6\% molar yield of 9-OH-4-HP [26]. Mycobacterium sp. VKM Ac1815D, Mycobacterium sp. VKM Ac-1817D, and Mycobacterium fortuitum ATCC-6842 have also been reported to accumulate a small amount of 9-OH-4-HP during the 9-OH-AD production process [4-6]. The enzymes that catalyse 22-hydroxy-3,24-dioxo-4-ene-cholest-COA into 4-HP homologues remain unidentified. Thus, Hsd4A is normally chosen to manipulate the metabolic flux to generate AD homologues or 4-HP homologues. Xu reported the characterization of Hsd4A in vivo and in vitro, testifying that deletion of $h s d 4 A$ resulted in blockage of the $C 19$ steroid pathway and enhanced the accumulation of 4-HP homologues. During the Hsd4A investigation, Xu constructed a 9-OH-4-HPproducing strain by knocking out $h s d 4 A$ in the $k s t D$-deficient strain of $M$. neoaurum ATCC 25795 . This mutant strain displayed $32 \%$ molar yield of $9-\mathrm{OH}-4-\mathrm{HP}$ and $15 \%$ molar yield of $9-\mathrm{OH}-\mathrm{AD}$ from $40 \mathrm{~g} \mathrm{~L}^{-1}$ phytosterols [3]. Here, in this research, it was confirmed that double knockout of $h s d 4 A$ and fadA5 could further block the $\mathrm{C} 19$ steroid pathway. The purity and molar yield of 9-OH-4-HP of strain $\Delta k s t D s \Delta h s d 4 A \triangle$ fadA5 were notably higher than those of Xu's strain. Although strain $\Delta k s t D s \Delta h s d 4 A \triangle$ fadA5 did not perform well when cultured with higher concentrations of phytosterols, the purity of 9-OH-4-HP was not influenced, indicating its potency as a promising 9-OH-4-HP producer.

AD homologues accumulated in the 9-OH-4-HP producer strains $\Delta k s t D s \Delta h s d 4 A, \Delta k s t D s \Delta$ fadA5, and $\Delta k s t D s \Delta h s d 4 A \triangle \mathrm{fad} A 5$, indicating incomplete blockage of the $C 19$ steroid pathway. Similar results have been previously reported. Analysis of the M. neoaurum CCTCC AB2019054 genome revealed that there were 6 proteins with $>38 \%$ identity and 10 proteins with $31-38 \%$ identity to Hsd $4 \mathrm{~A}$, which may compensate for its function [30]. The most identical gene, $h s d 4 A 2$ ( $45 \%$ identity to $h s d 4 A$ ), was deleted, 
and fermentation analysis revealed that it can indeed produce 4-HBC at a significantly increased ratio. A similar result was found in M. neoaurum DSM 44074. Eight proteins showed certain identities to Hsd4A, indicating the presence of isoenzymes of Hsd4A in the genome of M. neoaurum DSM 44074.

Some attempts have been made to enhance the ability of microorganisms to transform phytosterols. Considering the toxicity of phytosterols and their derivatives to cells, the balance of the intracellular environment could improve the ability of microorganisms to transform phytosterols. Intracellular factors such as NAD ${ }^{+}$and NADH have drawn increasing attention in recent years. NAD ${ }^{+}$and NADH participate in multiple steps during steroid bioconversion, and the intracellular $\mathrm{NAD}^{+}$concentration decreases as it is consumed. Regeneration of $\mathrm{NAD}^{+}$and enhancement of the NAD $/ \mathrm{NADH}$ ratio have been proven to be able to enhance the ability of microorganisms to transform phytosterols. Overexpression of NADH oxidase in $M$. neoaurum JC-12 increased ADD production by 43\% [17]. Overexpression of flavin oxidoreductase and NADH oxidase from Lactobacillus brevis in M. neoaurum TCCC 11978 increased the $\mathrm{NAD}^{+} / \mathrm{NADH}$ ratio by $113 \%$ and $192 \%$, respectively, and significantly enhanced the conversion ratio of $\mathrm{AD}(\mathrm{D})$ [18]. The type II NADH dehydrogenases $n d h N$ and $n d h F$ were overexpressed in $M$. neoaurum MNR, resulting in an increase in the $\mathrm{NAD}^{+} / \mathrm{NADH}$ ratio from 3.93 to 5.91 and 10.96 , respectively. The highest molar biotransformation rates of $A D$ with $5 \mathrm{~g} \mathrm{~L}^{-1}$ phytosterol feed were $5.32 \%$ and $12.38 \%$ higher than those of the original strain, respectively.

In addition, reactive oxygen species are generated during the conversion process of sterols, which impair cell viability and hinder the conversion of sterols to steroid synthons. Elimination of ROS and $\mathrm{H}_{2} \mathrm{O}_{2}$ have been reported to be an effective method to improve cell growth under phytosterol feed and enhance phytosterol bioconversion. Combinatorial augmentation with catalase, mycothiol, and ergothioneine increased 4-HP productivity by $47.5 \%$ in $M$. neoaurum WIII-egt\&msh\&cat [31]. Elimination of $\mathrm{H}_{2} \mathrm{O}_{2}$ in $M$. neoaurum JC-12 increased the 4-HP yield by $24 \%$. In this study, when combining the abilities of NAD ${ }^{+}$ regeneration and $\mathrm{H}_{2} \mathrm{O}_{2}$ elimination, the performance of the new mutant strain $\Delta k s t D s \Delta h s d 4 A \triangle$ fadA5-NK to transform phytosterols into 9-OH-4-HP improved. The extracellular $\mathrm{H}_{2} \mathrm{O}_{2}$ concentration of $\Delta k s t D s \Delta h s d 4 A \triangle$ fadA5-NK after culture with $5 \mathrm{~g} \mathrm{~L}^{-1}$ phytosterols remained at a low level, which was only $49.4 \%$ that of the extracellular $\mathrm{H}_{2} \mathrm{O}_{2}$ concentration of $\Delta k s t D s \Delta h s d 4 A \Delta f a d A 5$ culture under the same conditions. The $\mathrm{NAD}^{+} / \mathrm{NADH}$ ratio was also enhanced by $25.4 \%$ after $96 \mathrm{~h}$. Although the molar yield of $9-$ $\mathrm{OH}-4-\mathrm{HP}$ decreased as the phytosterol concentration increased, the molar yield of 9-0H-4-HP from strain $\Delta k s t D s \Delta h s d 4 A \triangle$ fadA5-NK was significantly higher than that from strain $\Delta k s t D s \Delta h s d 4 A \triangle$ fadA5 at the same phytosterol concentration. The highest yield of 9-OH-4-HP was $3.58 \mathrm{~g} \mathrm{~L}^{-1}$, which was achieved when $\Delta k s t D s \Delta h s d 4 A \Delta$ fadA5-NK was fed $5 \mathrm{~g} \mathrm{~L}^{-1}$ phytosterols. The molar yield of 9-0H-4-HP from strain $\Delta k s t D s \Delta h s d 4 A \triangle$ fadA5-NK was $28.7 \%$ higher than that from strain $\Delta k s t D s \Delta h s d 4 A \triangle$ fadA5 with $5 \mathrm{~g} \mathrm{~L}^{-1}$ phytosterol feeding. All of these results proved that the elimination of $\mathrm{H}_{2} \mathrm{O}_{2}$ and regeneration of $N A D^{+}$ could be an effective method to improve phytosterol bioconversion. 
When $10 \mathrm{~g} \mathrm{~L}^{-1}$ phytosterols were cultured with $\Delta k s t D s \Delta h s d 4 A \Delta$ fadA5-NK, the molar yield of 9-OH-4-HP was $32.6 \%$, indicating further manipulation to enhance its molar yield. A number of different aqueousorganic two-phase systems have been studied, such as water-vegetable oils and water-ionic liquids [32]. The use of cyclodextrins could enhance the uptake of phytosterols by microorganisms [16, 33]. Recently, some novel methods have been developed to improve phytosterol bioconversion. Increasing cell permeability could improve the production of phytosterol metabolites [34, 35]. Deletion of the transmembrane transporter trehalose monomycolate $m m p L 3$ in a 4-HP-producing strain derived from $M$. neoaurum ATCC 25795 increased 4-HP production by $24.7 \%$. A "resting cell-cyclodextrin" system has also been widely used in industry and research to improve bioconversion ability.

\section{Methods}

\section{Bacterial strains, plasmids, medium, and reagents}

The stains and plasmids used in this study are described in Table 1. E. coli DH5a stored in the laboratory was used for plasmid amplification. Wild-type M. neoaurum DSM 44074 (DSM 44704) was purchased from Deutsche Sammlung von Mikroorganismenund Zellkulturen (DSMZ, GERMANY). All other strains were derived from M. neoaurum DSM 44704. Common plasmids and primers (Table S1) were used to construct the mutants. E. coli DH5a was cultured at $37^{\circ} \mathrm{C}$ and $200 \mathrm{rpm}$ in $50 \mathrm{~mL}$ of Luria-Bertani (LB) medium ( $10 \mathrm{~g} \mathrm{~L}^{-1}$ tryptone, $10 \mathrm{~g} \mathrm{~L}^{-1} \mathrm{NaCl}, 5 \mathrm{~g} \mathrm{~L}^{-1}$ yeast extracts, $\mathrm{pH}$ 7.0). Mycobacterium cells were cultured in MYD medium $\left(0.6 \mathrm{~g} \mathrm{~L}^{-1} \mathrm{~K}_{2} \mathrm{HPO}_{4} \cdot 3 \mathrm{H}_{2} \mathrm{O}, 5.4 \mathrm{~g} \mathrm{~L}^{-1} \mathrm{NaNO}_{3}, 6 \mathrm{~g} \mathrm{~L}^{-1}\right.$ glucose, $15 \mathrm{~g} \mathrm{~L}^{-1}$ yeast extract and an initial $\mathrm{pH}$ value 7.5) and fermented with MP01 medium $\left(10 \mathrm{~g} \mathrm{~L}^{-1}\right.$ corn steep powder, $20 \mathrm{~g} \mathrm{~L}^{-1}$ glucose, $2 \mathrm{~g} \mathrm{~L}^{-1} \mathrm{~K}_{2} \mathrm{HPO}_{4} \cdot 3 \mathrm{H}_{2} \mathrm{O}, 1.0 \mathrm{~g} \mathrm{~L}^{-1} \mathrm{MgSO}_{4} \cdot 7 \mathrm{H}_{2} \mathrm{O}, 2.0 \mathrm{~g} \mathrm{~L}^{-1} \mathrm{NaNO}_{3}, 2 \%$ Tween $80(\mathrm{v} / \mathrm{v})$, and an initial $\mathrm{pH}$ value 7.5 ) at $30^{\circ} \mathrm{C}$ and $200 \mathrm{rpm}$.

The phytosterols consisted of $45 \% \beta$-sitosterol, $37 \%$ campesterol, and $18 \%$ stigmasterol, which were purchased from Yunnan Biological Products Co., Ltd. (Yunnan, China). AD and 9-OH-AD were obtained from Shanghai Macklin Biochemical Co., Ltd. (China). (2-Hydroxypropyl)- $\beta$-cyclodextrin (HP- $\beta$-CD) was purchased from Zhiyuan Biotechnology Co., Ltd. (Shandong, China). The ClonExpress $₫$ One Step Cloning Kit was purchased from Vazyme Biotech Co., Ltd. (Nanjing, China). The Hydrogen Peroxide $\left(\mathrm{H}_{2} \mathrm{O}_{2}\right)$ Content Assay Kit and Nicotinamide Adenine Dinucleotide NAD(H) Content Assay Kit were obtained from Sangon Biotech Co., Ltd. (Shanghai, China).

\section{Bioinformatic analysis}

The genome of M. neoaurum DSM 44074 was sequenced by Shanghai Majorbio Co., Ltd. The DNA sample was extracted and sheared into 400-500 bp fragments using a Covaris M220 Focused Acoustic Shearer (Covaris, USA). Illumina sequencing libraries were prepared from the sheared fragments using a NEXTflex ${ }^{\text {TM }}$ Rapid DNS-Seq Kit (Bioo Scientific, USA). The sequencing data were assembled using SOAPdenovo2(GitHub - aquaskyline/SOAPdenovo2: Next generation sequencing reads de novo 
assembler.). Further prediction and annotation were produced by Glimmer (Glimmer (jhu.edu)) and BLAST (blast.ncbi.nlm.nih.gov). The putative genes for $k s t D$, $h s d 4 A$, and fadA5 were identified by comparison with known gene sequences taken from the NCBI database. MEGA-X software (Home (megasoftware.net)) was used to construct a phylogenetic tree of $h s d 4 A$ and fadA5 with the known amino acid sequences taken from the NCBI.

\section{Mutant strain construction}

A CRISPR-assisted nonhomologous end-joining strategy was used to delete the target gene in M. neoaurum DSM 44074 based on previous reports. The PSBY1 plasmid harbouring cpf1 was obtained from Jiang [36], and the PCR-Hyg plasmid harbouring sgRNA was obtained from Sun [37].

ClonExpress $\mathbb{O}$ One Step Cloning Kit mutated spacers were used to construct different plasmids harbouring target sgRNA. The plasmid harbouring target sgRNA was transfected into M. neoaurum, and the PSBY1 plasmid was transfected beforehand by electroporation. The recombinant clones were sequenced using specific primers to determine the deletion.

The vector P38Mu (pMV306 with the Psmyc promoter) with kanamycin resistance was used to overexpress the target gene. The genes $h s d 4 A$, fadA5, katE from M.neoaurum DSM 44074, and nox from Bacillus subtilis were recombined on P38Mu. Specific primers were used to amplify the corresponding gene, and the PCR product was inserted into the Nde区 site (and Hind site, if two genes were inserted) of P38Mu using the ClonExpress $₫$ One Step Cloning Kit.

\section{Bioconversion and analysis}

The transformation capability of the mutant strains was identified in MP01 medium with an initial phytosterol concentration of $1 \mathrm{~g} \mathrm{~L}^{-1}$. A concentration gradient was later tested to further determine the capability of phytosterol bioconversion. Phytosterols were prepared in (2-hydroxypropyl)- $\beta$-cyclodextrin $(\mathrm{HP}-\beta-\mathrm{CD})$ at a ratio of 1:1.5. The recombinant cells were inoculated into $30 \mathrm{~mL}$ of MYD medium in a 250 $\mathrm{mL}$ shaker flask and cultured at $30^{\circ} \mathrm{C}$ and $200 \mathrm{rpm}$. Three millilitres of seed medium was transferred to 30 $\mathrm{mL}$ of MP01 medium in a $250 \mathrm{~mL}$ shaker flask with a baffle when the optical density reached the mid-log exponential phase. The fermentation of $M$. neoaurum DSM 44074 and recombinant strains was sampled every 12 or $24 \mathrm{~h}$, and three replicates were used to measure the steroids. The bioconversion mixture was extracted with 3 volumes of ethyl acetate, and the solvent was removed to give a residue that was redissolved in methanol. The resulting solution was used for HPLC analysis. HPLC was performed on a Shimadzu Separations module connected to a Shimadzu SPD-M20A detector equipped with a C18 column $(250 \mathrm{~mm} \times 4.6 \mathrm{~mm}, 5 \mu \mathrm{m})$ and detected at a wavelength of $254 \mathrm{~nm}$. A mixture of methanol and water $(80: 20, v / v)$ was used as the mobile phase at a flow rate of $0.8 \mathrm{~mL} \mathrm{~min}^{-1}$.

Extracellular $\mathrm{H}_{2} \mathrm{O}_{2}$ concentrations were measured according to the operating manual of the Hydrogen Peroxide $\left(\mathrm{H}_{2} \mathrm{O}_{2}\right)$ Content Assay Kit. NADH and NAD intracellular concentrations were measured 
according to the operating manual of the Nicotinamide Adenine Dinucleotide, NAD $(H)$ Content Assay Kit.

The accumulation of 9-OH-4-HP was achieved by blocking the $\mathrm{C} 19$ steroid pathway and 3-ketosteroid- $\Delta^{1}$ dehydrogenation. Compared with single deletion of $h s d 4 A$ and single deletion of fadA5, double deletion of $h s d 4 A$ and fadA5 could further block the $\mathrm{C} 19$ steroid pathway. By eliminating $\mathrm{H}_{2} \mathrm{O}_{2}$ and regenerating $\mathrm{NAD}^{+}$in the triple $h s d 4 A$, fadA5, and kstDs knockout strain, the highest 9-OH-4-HP productivity was $3.58 \mathrm{~g}$ $\mathrm{L}^{-1}$ with $5 \mathrm{~g} \mathrm{~L}^{-1}$ phytosterol feed.

\section{Abbreviations}

AD: androst-4-ene-3,17-dione; ADD: androst-1,4-diene-3,17-dione; 9-OH-AD: 9-hydroxy-androst-4-ene-3,17dione; 4-HP: 21-hydroxy-20-methyl-pregna-4-en-3-one; 1,4-HP: 21-hydroxy-20-methyl-pregna-1,4-dien-3-one; 9-OH-4-HP: 9,21-dihydroxy-20-methyl-pregna-4-en-3-one; KstDs: 3-ketosteroid- $\Delta^{1}$-dehydrogenases; KSHs: 9a-hydroxylase; HPs: C22 steroids; Hsd4A: $\beta$-hydroxyacyl-CoA dehydrogenase; FadA5: acyl-COA thiolase; ROS: Reactive oxygen species; $\mathrm{H}_{2} \mathrm{O}_{2}$ : hydrogen peroxide; $\mathrm{NAD}^{+} / \mathrm{NADH}$ : nicotinamide adenine dinucleotides; NOX: NADH oxidase; KatE: catalase; HPLC: high performance liquid chromatography

\section{Declarations}

\section{Ethics approval and consent to participate}

Not applicable.

\section{Consent for publication}

Not applicable.

\section{Availability of data and materials}

All data generated and analyzed during this study are included in this published article and its additional files.

\section{Author's contributions}

BGZ and YCY designed the study. YCY carried out the gene knockout and overexpression. YCY and ZGM performed the phytosterol bioconversion. YCY, XCL, GLD and JXZ analyzed the data. YCY wrote the manuscript. BGZ, JSS and JPS reviewed the manuscript.

All authors read and approved the final manuscript. 


\section{Competing interests}

The authors declare no conflict of interest

\section{Funding}

This research was funded by National Key R\&D Program of China, grant number 2017 YFE0112700.

\section{Acknowledgement}

We sincerely thank Yu Jiang (CAS Center for Excellence in Molecular Plant Sciences Institute of Plant Physiology and Ecology, Chinese Academy of Sciences, Shanghai, China) for providing the plasmid PSBY1 and Yicheng Sun (Chinese Academy of Medical Sciences and Peking Union Medical College, Beijing, China) for providing the plasmid Pcr-Hyg. We also thank W.R. Jacobs Jr. (Howard Hughes Medical Institute) for providing the plasmids pMV306

\section{References}

1. Finocchi C, Ferrari M. Female reproductive steroids and neuronal excitability. Neurol Sci. 2011;32(Suppl 1):S31-5.

2. Rugutt JK, Rugutt KJ. Antimycobacterial activity of steroids, long-chain alcohols and lytic peptides. Nat Prod Res. 2012;26(11):1004-11.

3. Xu LQ, et al. Unraveling and engineering the production of 23,24-bisnorcholenic steroids in sterol metabolism. Rep. 2016;6:21928.

4. Donova MV, et al. Mycobacterium sp. mutant strain producing 9alpha-hydroxyandrostenedione from sitosterol. Appl Microbiol Biotechnol. 2005;67(5):671-8.

5. Shtratnikova VY, et al. Effect of methyl-beta-cyclodextrin on gene expression in microbial conversion of phytosterol. Appl Microbiol Biotechnol. 2017;101(11):4659-67.

6. Wovcha MG, et al. Bioconversion of sitosterol to useful steroidal intermediates by mutants of Mycobacterium fortuitum. Biochim Biophys Acta. 1978;531(3):308-21.

7. Yao K, et al. Identification and engineering of cholesterol oxidases involved in the initial step of sterols catabolism in Mycobacterium neoaurum. Metab Eng. 2013;15:75-87.

8. Brzostek A, et al. Cholesterol oxidase is required for virulence of Mycobacterium tuberculosis. FEMS Microbiol Lett. 2007;275(1):106-12.

9. Petrusma M, et al. Multiplicity of 3-Ketosteroid-9alpha-Hydroxylase enzymes in Rhodococcus rhodochrous DSM43269 for specific degradation of different classes of steroids. J Bacteriol. 2011;193(15):3931-40. 
10. Van, d.G., R., et al., Targeted disruption of the kstD gene encoding a 3-ketosteroid delta(1)dehydrogenase isoenzyme of Rhodococcus erythropolis strain SQ1. Applied \& Environmental Microbiology, 2000. 66(5): p. 2029-36.

11. Sedlaczek L, Smith LL. Biotransformations of steroids. Crit Rev Biotechnol. 1988;7(3):187-236.

12. Yao $\mathrm{K}$, et al. Characterization and engineering of 3-ketosteroid- $\triangle 1$-dehydrogenase and 3-ketosteroid9a-hydroxylase in Mycobacterium neoaurum ATCC 25795 to produce 9a-hydroxy-4-androstene-3,17dione through the catabolism of sterols. Metab Eng. 2014;24:181-91.

13. Yang, et al. The effect of 3-ketosteroid- $\Delta 1$-dehydrogenase isoenzymes on the transformation of $A D$ to $9 \mathrm{a}-\mathrm{OH}-\mathrm{AD}$ by Rhodococcus rhodochrous DSM43269. Journal of Industrial Microbiology Biotechnology. 2016;43(9):1303-11.

14. Sun $\mathrm{H}$, et al. Enhancing production of 9a-hydroxy-androst-4-ene-3,17-dione (9-OHAD) from phytosterols by metabolic pathway engineering of mycobacteria. Chem Eng Sci. 2021;230(47):116195.

15. Nesbitt NM, et al. A thiolase of Mycobacterium tuberculosis is required for virulence and production of androstenedione and androstadienedione from cholesterol. Infect Immun. 2010;78(1):275-82.

16. Su L, et al., The Sterol Carrier Hydroxypropyl-beta-Cyclodextrin Enhances the Metabolism of Phytosterols by Mycobacterium neoaurum. Appl Environ Microbiol, 2020. 86(15).

17. Shao M, et al., Intracellular Environment Improvement of Mycobacterium neoaurum for Enhancing Androst-1,4-Diene-3,17-Dione Production by Manipulating NADH and Reactive Oxygen Species Levels. Molecules, 2019. 24(21).

18. Su LQ, et al., Cofactor engineering to regulate $N A D(+) / N A D H$ ratio with its application to phytosterols biotransformation. Microbial Cell Factories, 2017. 16.

19. Zhou XL, et al. Efficient production of androstenedione by repeated batch fermentation in waste cooking oil media through regulating $\mathrm{NAD}(+) / \mathrm{NADH}$ ratio and strengthening cell vitality of Mycobacterium neoaurum. Biores Technol. 2019;279:209-17.

20. Orrego R, et al. Pulp and Paper Mill Effluent Treatments Have Differential Endocrine-Disrupting Effects on Rainbow Trout. Environ Toxicol Chem. 2009;28(1):181-8.

21. Nieminen $P$, et al. Phytosterols act as endocrine and metabolic disruptors in the European polecat (Mustela putorius). Toxicol Appl Pharmacol. 2002;178(1):22-8.

22. Denton TE, et al. Masculinization of female mosquitofish by exposure to plant sterols and Mycobacterium smegmatis. Bull Environ Contam Toxicol. 1985;35(5):627-32.

23. Li JY, et al. Crystal-Structure of Cholesterol Oxidase Complexed with a Steroid Substrate Implications for Flavin Adenine-Dinucleotide Dependent Alcohol Oxidases. Biochemistry. 1993;32(43):11507-15.

24. Uhia I, et al. Initial step in the catabolism of cholesterol by Mycobacterium smegmatis mc2155. Environ Microbiol. 2011;13(4):943-59. 
25. Ezraty B, et al. Oxidative stress, protein damage and repair in bacteria. Nat Rev Microbiol. 2017;15(7):385-96.

26. Donova MV, et al. Mycobacterium sp. mutant strain producing 9a-hydroxyandrostenedione from sitosterol. Applied Microbiology Biotechnology. 2005;67(5):671-8.

27. Engineered 3-Ketosteroid 9a-Hydroxylases in Mycobacterium neoaurum: an Efficient Platform for Production of Steroid Drugs. Applied and Environmental Microbiology, 2018. 84(14): p. e02777-17.

28. Heras LFdL, et al. Molecular characterization of three 3-ketosteroid- $\Delta$ (1)-dehydrogenase isoenzymes of Rhodococcus ruber strain Chol-4. J Steroid Biochem Mol Biol. 2012;132(3-5):271-81.

29. Mathieu JM, et al. 7-Ketocholesterol Catabolism by Rhodococcus jostii RHA1. Applied Environmental Microbiology. 2010;76(1):352-5.

30. Peng $\mathrm{H}$, et al. A Dual Role Reductase from Phytosterols Catabolism Enables the Efficient Production of Valuable Steroid Precursors. Angew Chem Int Ed Engl. 2021;60(10):5414-20.

31. Sun WJ, et al. Characterization and engineering control of the effects of reactive oxygen species on the conversion of sterols to steroid synthons in Mycobacterium neoaurum. Metab Eng. 2019;56:97110.

32. Yuan JJ, et al. Side-chain cleavage of phytosterols by Mycobacterium sp MB 3683 in a biphasic ionic liquid/aqueous system. J Chem Technol Biotechnol. 2016;91(10):2631-7.

33. Shen $Y$, et al. Cyclic utilization of HP- $\beta$-CD in the bioconversion of cortisone acetate by Arthrobacter simplex. Biotech Lett. 2016;38(4):597-602.

34. Xiong LB, et al., Improving the production of 22-hydroxy-23,24-bisnorchol-4-ene-3-one from sterols in Mycobacterium neoaurum by increasing cell permeability and modifying multiple genes. Microbial Cell Factories, 2017. 16.

35. Xiong LB, et al., Enhancing the bioconversion of phytosterols to steroidal intermediates by the deficiency of kasB in the cell wall synthesis of Mycobacterium neoaurum. Microbial Cell Factories, 2020. 19(1).

36. Sun BB, et al., A CRISPR-Cpf1-Assisted Non-Homologous End Joining Genome Editing System of Mycobacterium smegmatis. Biotechnology Journal, 2018. 13(9).

37. Yan MY, et al., CRISPR-Cas12a-Assisted Recombineering in Bacteria. Applied and Environmental Microbiology, 2017. 83(17).

38. Stover CK, et al. New Use of Bcg for Recombinant Vaccines. Nature. 1991;351(6326):456-60.

39. Liu XC, et al., Biotransformation of Phytosterols to Androst-1,4-Diene-3,17-Dione byMycobacteriumsp. ZFZ Expressing 3-Ketosteroid-Delta(1)-Dehydrogenase. Catalysts, 2020. 10(6).

\section{Tables}

Table 1

Strains and plasmids used in this study 


\begin{tabular}{|c|c|c|}
\hline Name & Description & Source \\
\hline \multicolumn{3}{|l|}{ Strains } \\
\hline $\begin{array}{l}\text { M. neoaurum DSM } \\
44704\end{array}$ & $\begin{array}{l}\text { Wild type strain, Sterol consumer with no detectable } \\
\text { intermediates }\end{array}$ & \\
\hline$\Delta k s t D$ & kstD1, kstD2, and $k s t D 3$ deleted in M. neoaurum DSM 44704 & $\begin{array}{l}\text { This } \\
\text { study }\end{array}$ \\
\hline$\Delta k s t D \Delta h s d 4 A$ & $h s d 4 A$ deleted in $\Delta k s t D$ strain & $\begin{array}{l}\text { This } \\
\text { study }\end{array}$ \\
\hline$\Delta k s t D \Delta$ fadA5 & fad $A 5$ deleted in $\triangle K s t D$ strain & $\begin{array}{l}\text { This } \\
\text { study }\end{array}$ \\
\hline$\Delta k s t D \Delta h s d 4 A-h s d 4 A$ & $\Delta k s t D \Delta h s d 4 A$ strain harboring $\mathrm{P} 38 \mathrm{Mu}-\mathrm{H} s \mathrm{~d} 4 \mathrm{~A}$ & $\begin{array}{l}\text { This } \\
\text { study }\end{array}$ \\
\hline$\Delta k s t D \triangle$ fadA5-fadA5 & $\Delta k s t D \triangle$ fadA5 strain harboring P38Mu-FadA5 & $\begin{array}{l}\text { This } \\
\text { study }\end{array}$ \\
\hline$\Delta k s t D \Delta h s d 4 A \Delta$ fadA5 & $h s d 4 A$ and $f a d A 5$ deleted in $\triangle k s t D$ strain & $\begin{array}{l}\text { This } \\
\text { study }\end{array}$ \\
\hline $\begin{array}{l}\Delta k s t D \Delta h s d 4 A \Delta \text { fadA5- } \\
\text { NK }\end{array}$ & $\Delta k s t D \Delta h s d 4 A \Delta$ fadA5strain harboring P38Mu-NK & $\begin{array}{l}\text { This } \\
\text { study }\end{array}$ \\
\hline \multicolumn{3}{|l|}{ Plasmids } \\
\hline PSBY1 & $\begin{array}{l}\text { Derived from pMV261 and contains FnCpf1 C. glutamicum } \\
\text { codon-optimized; KanR }\end{array}$ & [36] \\
\hline PCR-Hyg & Plasmid for sgRNA production & [37] \\
\hline Pam-KstD1 & PCR-Hyg containing $k s t D 1$ spacer & $\begin{array}{l}\text { This } \\
\text { study }\end{array}$ \\
\hline Pam-KstD2 & PCR-Hyg containing $k s t D 2$ spacer & $\begin{array}{l}\text { This } \\
\text { study }\end{array}$ \\
\hline Pam-KstD3 & PCR-Hyg containing $k s t D 3$ spacer & $\begin{array}{l}\text { This } \\
\text { study }\end{array}$ \\
\hline Pam-Hsd4A & PCR-Hyg containing $h s d 4 A$ spacer & $\begin{array}{l}\text { This } \\
\text { study }\end{array}$ \\
\hline Pam-FadA5 & PCR-Hyg containing fadA5 spacer & $\begin{array}{l}\text { This } \\
\text { study }\end{array}$ \\
\hline pMV306 & Mycobacterium integrative vector without promoter, $\operatorname{kan}^{\mathrm{R}}$ & [38] \\
\hline $\mathrm{P} 38 \mathrm{Mu}$ & pMV306 with Psmyc promoter, $\mathrm{Kan}^{\mathrm{R}}$ & [39] \\
\hline P38Mu-Hsd4A & Recombinant P38Mu for expression of $h s d 4 A$ & $\begin{array}{l}\text { This } \\
\text { study }\end{array}$ \\
\hline P38Mu-FadA5 & $\begin{array}{l}\text { Recombinant P38Mu for expression of fadA5 } \\
\text { Page } 17 / 23\end{array}$ & This \\
\hline
\end{tabular}


Table. 2

Relative production purity of M.neoaurum DSM 44074 and its derivative strains

\begin{tabular}{lllll} 
strain & \multicolumn{4}{l}{ Relative Purity (\%) } \\
& $9-\mathrm{OH}-\mathrm{AD}$ & $9-\mathrm{OH}-4-\mathrm{HP}$ & $\mathrm{AD}$ & Others \\
\hline DSM 44704 & 0 & 0 & 0 & 0 \\
\hline$\Delta k s t D$ & $90.4 \pm 3.1$ & $5.6 \pm 2.5$ & $1.9 \pm 0.2$ & $2.1 \pm 0.8$ \\
\hline$\Delta k s t D \Delta h s d 4 A$ & $7.2 \pm 2.2$ & $88.6 \pm 1.3$ & $2.4 \pm 0.5$ & $1.8 \pm 1.3$ \\
\hline$\Delta k s t D \Delta$ fadA5 & $8.0 \pm 1.9$ & $86.0 \pm 3.5$ & $3.3 \pm 0.4$ & $2.7 \pm 0.5$ \\
\hline$\Delta k s t D \Delta h s d 4 A-h s d 4 A$ & $90.0 \pm 2.2$ & $5.2 \pm 1.7$ & $1.8 \pm 0.2$ & $3.0 \pm 0.3$ \\
\hline$\Delta k s t D \Delta$ fadA5-fadA5 & $88.5 \pm 3.6$ & $4.4 \pm 2.3$ & $1.3 \pm 0.1$ & $5.8 \pm 1.2$ \\
\hline$\Delta k s t D \Delta h s d 4 A \Delta$ fadA5 & $2.0 \pm 1.1$ & $94.9 \pm 1.2$ & $0.3 \pm 0.1$ & $2.8 \pm 0.3$ \\
\hline$\Delta k s t D \Delta h s d 4 A \Delta$ fadA5-NK & $2.0 \pm 1.1$ & $97.0 \pm 1.1$ & $0.3 \pm 0.1$ & $0.7 \pm 0.3$
\end{tabular}

Table. 3

Maximum yield and molar yield of 9-OH-4-HP from $\Delta k s t D \Delta h s d 4 A \Delta$ fadA5 and $\Delta k s t D \Delta h s d 4 A \Delta$ fadA5-NK

\begin{tabular}{|c|c|c|c|c|}
\hline phytosterols & $\Delta k s t D \Delta h s d 4 A \Delta t$ & $d A 5$ & $\Delta k s t D \Delta h s d 4 A \Delta$ & $d A 5-N K$ \\
\hline$\left(\mathrm{g} \mathrm{L}^{-1}\right)$ & $\begin{array}{l}\text { Maximum yield } \\
\left(\mathrm{g} \mathrm{L}^{-1}\right)\end{array}$ & $\begin{array}{l}\text { Maximum molar } \\
\text { yield \%) }\end{array}$ & $\begin{array}{l}\text { Maximum yield } \\
\left(\mathrm{g} \mathrm{L}^{-1}\right)\end{array}$ & $\begin{array}{l}\text { Maximum molar } \\
\text { yield (\%) }\end{array}$ \\
\hline 1 & $0.66 \pm 0.02$ & $78.9 \pm 2.4$ & $0.68 \pm 0.04$ & $81.2 \pm 4.8$ \\
\hline 2 & $1.43 \pm 0.06$ & $84.8 \pm 3.6$ & $1.53 \pm 0.06$ & $90.8 \pm 3.6$ \\
\hline 5 & $2.78 \pm 0.11$ & $66.4 \pm 2.6$ & $3.58 \pm 0.15$ & $85.5 \pm 3.6$ \\
\hline 8 & $1.98 \pm 0.08$ & $31.5 \pm 1.2$ & $2.51 \pm 0.14$ & $38.5 \pm 2.1$ \\
\hline 10 & $1.73 \pm 0.09$ & $20.7 \pm 1.1$ & $2.73 \pm 0.19$ & $32.6 \pm 1.1$ \\
\hline
\end{tabular}




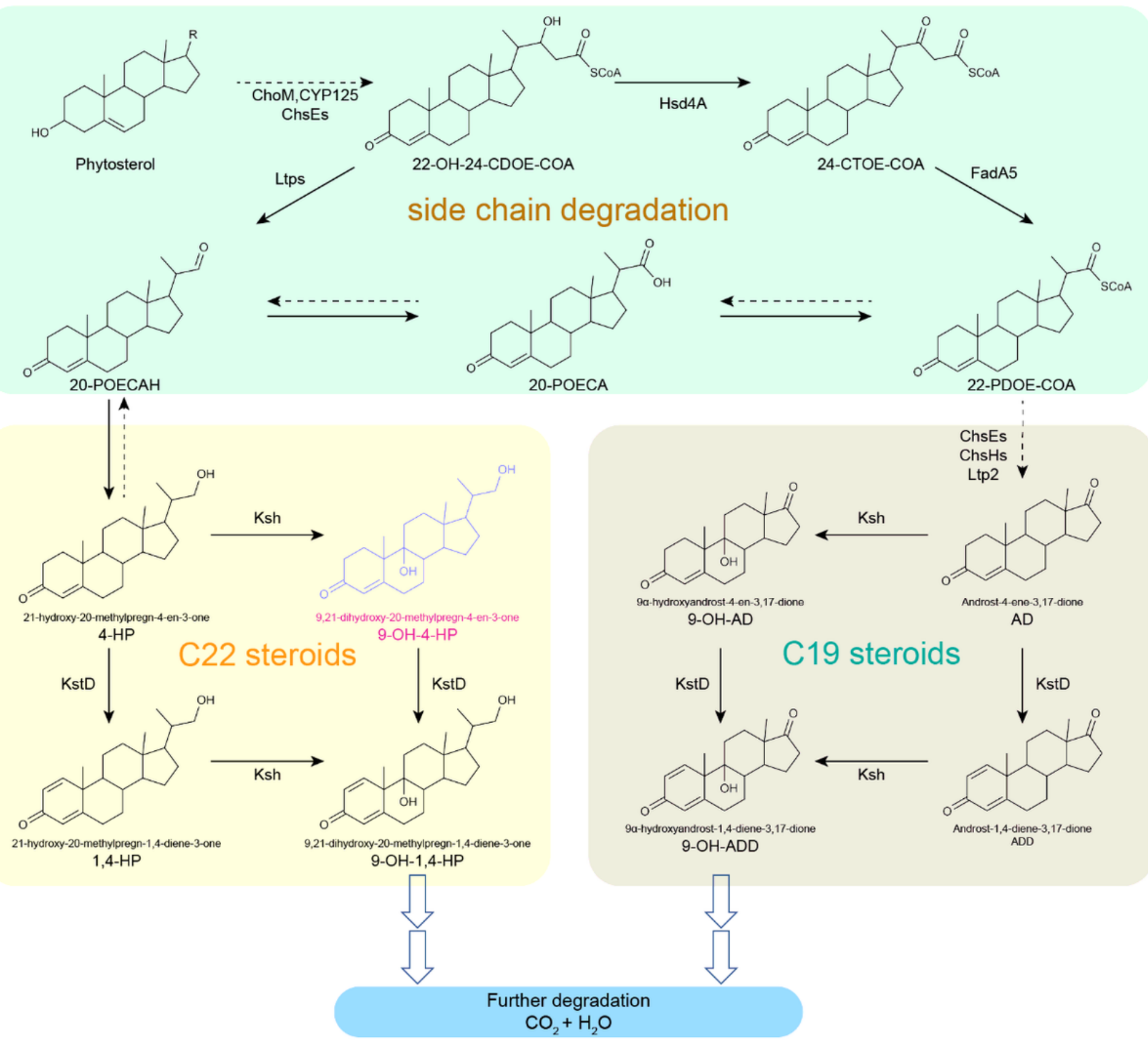

Figure 1

A schematic diagram of physterols side chain degradation in Mycobacterium. ChoM, Cholesterol oxidase; CYP125, cytochrome P450 125; ChsEs, acyl-CoA dehydrogenases; ChsHs, 3-oxo-23,24-bisnorchol4,17(20)-dien-22-oyl-CoA-hydratase; Ltp2, lipid transfer protein 2; Hsd4A, 17ß-hydroxysteroid dehydrogenase / $\beta$-hydroxyacyl-CoA dehydrogenase; FadA5, acetyl-CoA acetyltransferase/thiolase; KstD, 3-ketosteroid- $\triangle 1$-dehydrogenase; $\mathrm{KSH}$, 3-ketosteroid-9a-hydroxylase; 22-OH-24-CDOE-COA, 22-hydroxy3,24-dioxo-4-ene-cholest-COA; 24-CTOE-COA, 3,22,24-trioxo-4-ene-cholest-COA; 22-PDOE-COA, 3,22-dioxo4-ene-pregna-COA; 20-POECA, 3-oxo-4-ene-pregna-20-carboxylic acid; 20-POECAH, 3-oxo-4-ene-pregna-20carboxyaldehyde. 

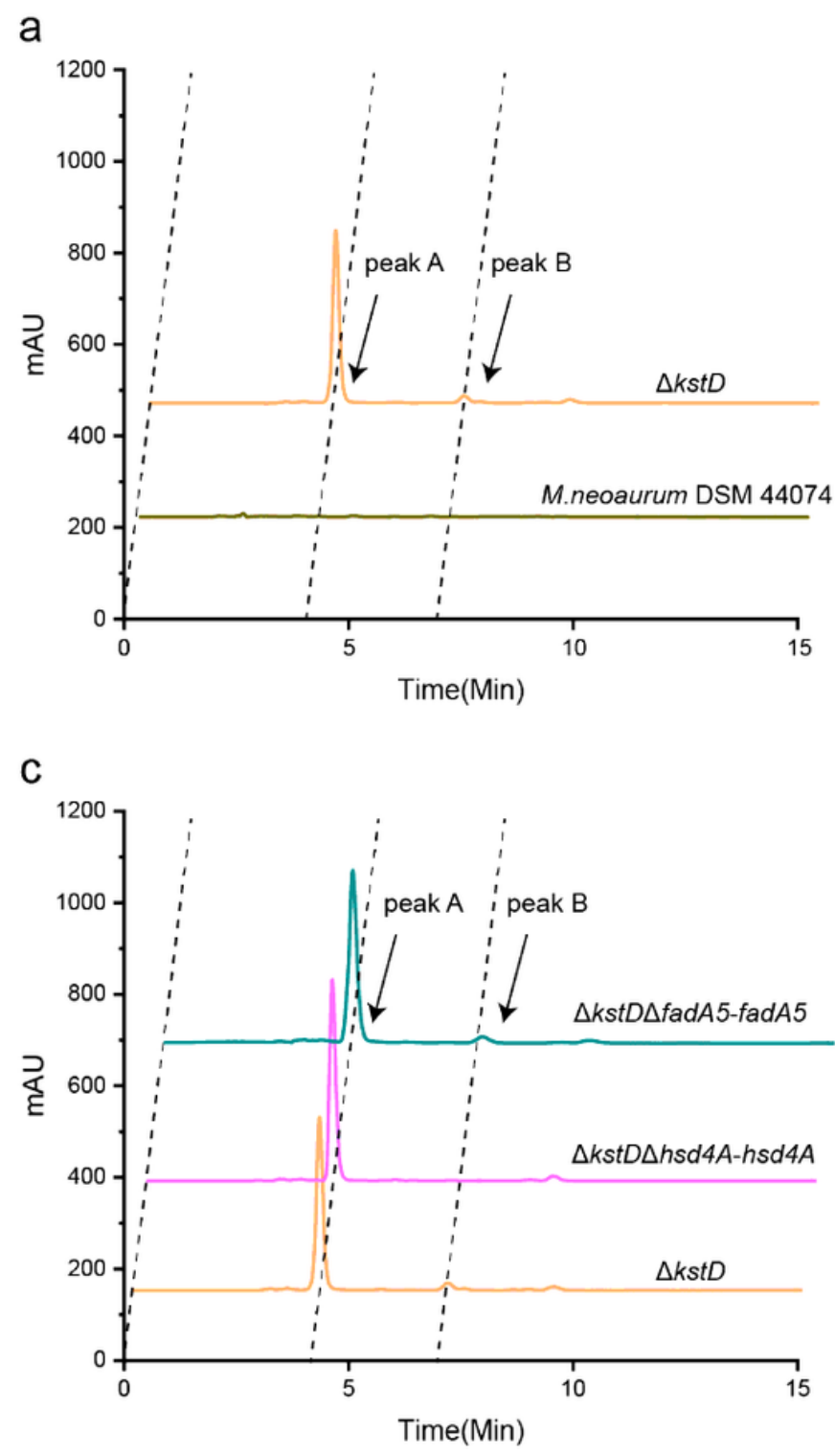

b

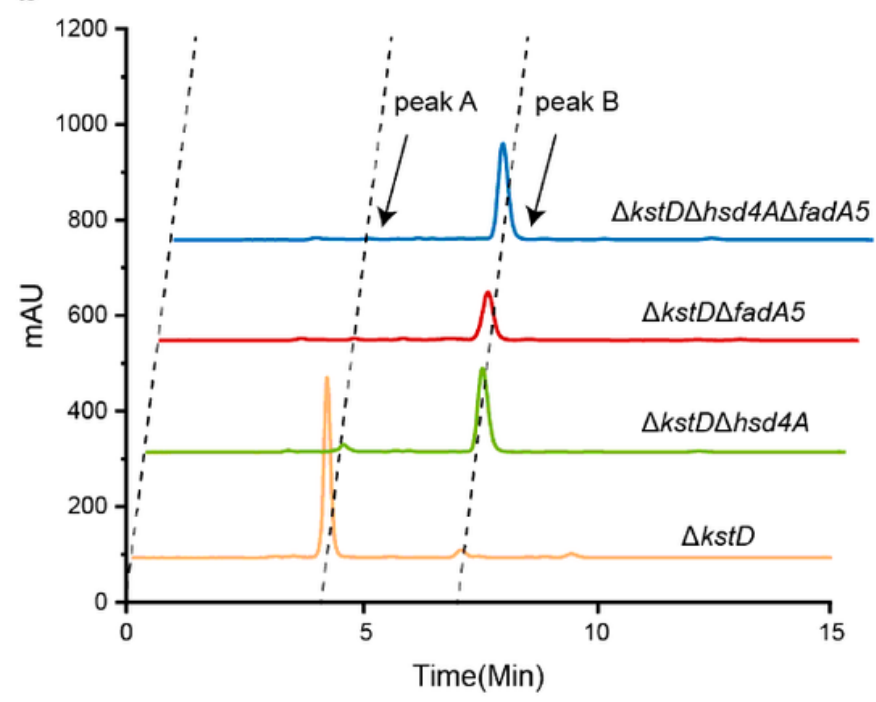

d
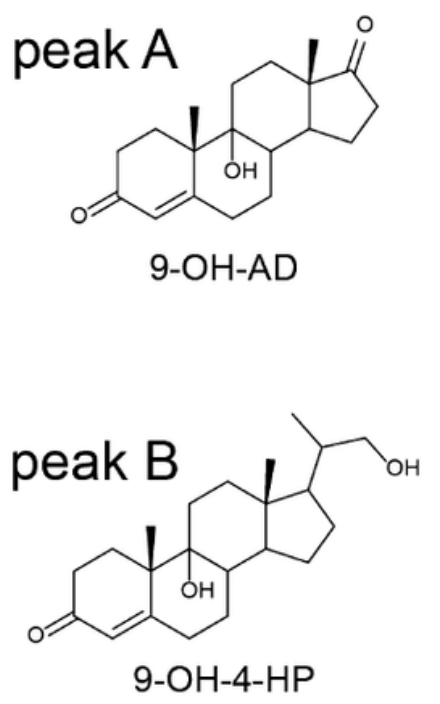

Figure 2

Phenotypic analyses of the metabolites of phytosterol by M.neoaurum DSM 44074 and its derivative strains. Peak A, 9-OH-AD; Peak B, 9-OH-4-HP. (a) HPLC chromatogram comparison of the products of M.neoarum DSM 44704 and $\triangle \mathrm{kstD}$ with $1 \mathrm{~g} \mathrm{~L}-1$ phytosterols feed. (b) HPLC chromatogram comparison of the products of $\Delta \mathrm{kstD}, \Delta \mathrm{kstD} \Delta \mathrm{hsd} 4 \mathrm{~A}, \Delta \mathrm{kstD} \Delta \mathrm{fadA} 5$ and $\Delta \mathrm{kstD} \Delta \mathrm{hsd} 4 \mathrm{~A} \Delta \mathrm{fadA} 5$ with $1 \mathrm{~g} \mathrm{~L}-1$ phytosterols feed. (3) HPLC chromatogram comparison of the products of $\triangle \mathrm{kstD}$, hsd4A complement strain $\Delta \mathrm{kstD} \Delta \mathrm{hsd} 4 \mathrm{~A}-\mathrm{hsd} 4 \mathrm{~A}$ and fadA5 complement strain $\Delta \mathrm{kstD} \Delta$ fadA5-fadA5, with $1 \mathrm{~g} \mathrm{L-1}$ phytosterols feed. (d) structure of peak A, 9-OH-AD, and peak B, 9-OH-4-HP. 

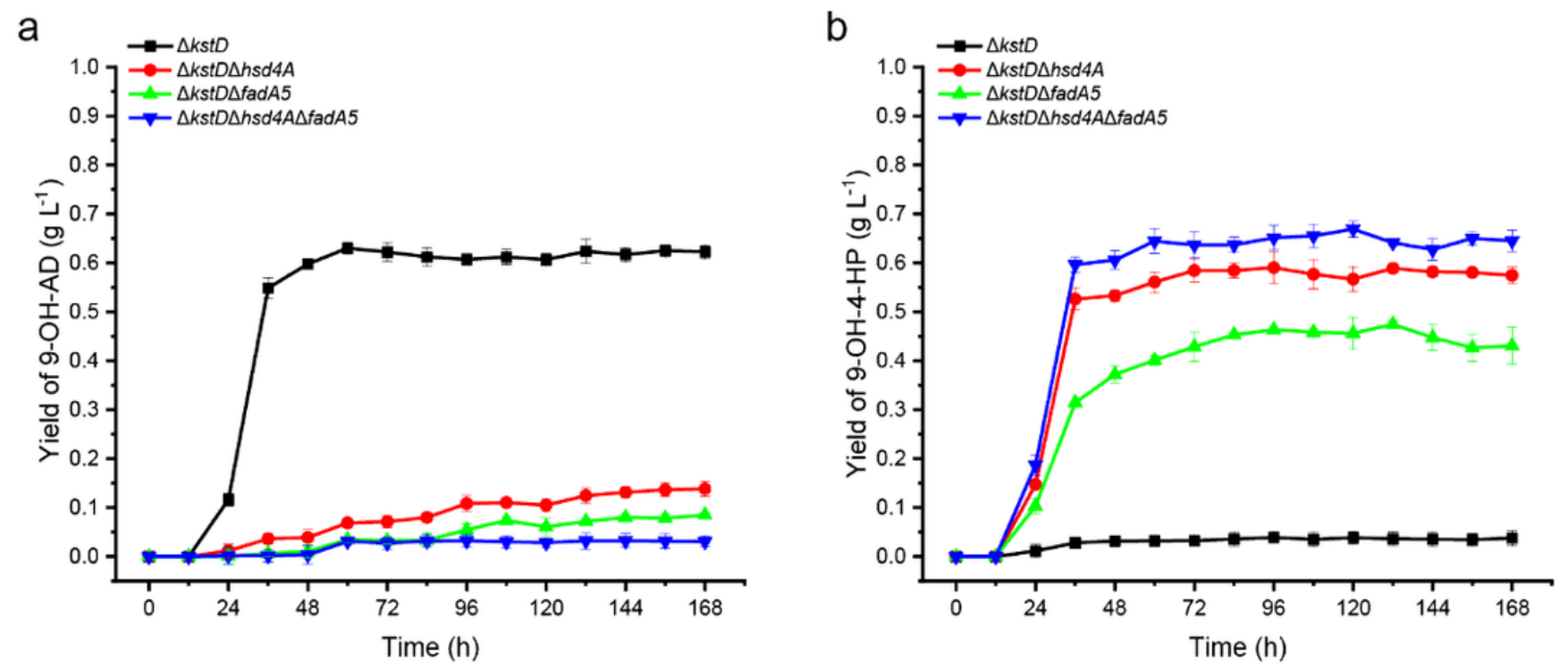

\section{Figure 3}

9-hydroxy steroids accumulation from $1 \mathrm{~g} \mathrm{L-1}$ phytosterols. (a) Time course of 9-OH-AD accumulation; (b) Time course of 9-OH-4-HP accumulation; single deletion of hsd4A or fadA5 caused increasement of 9-OH4-HP, and double deletion of hsd $4 \mathrm{~A}$ and fadA5 could obviously increase the productivity and selectivity of 9-OH-4-HP.
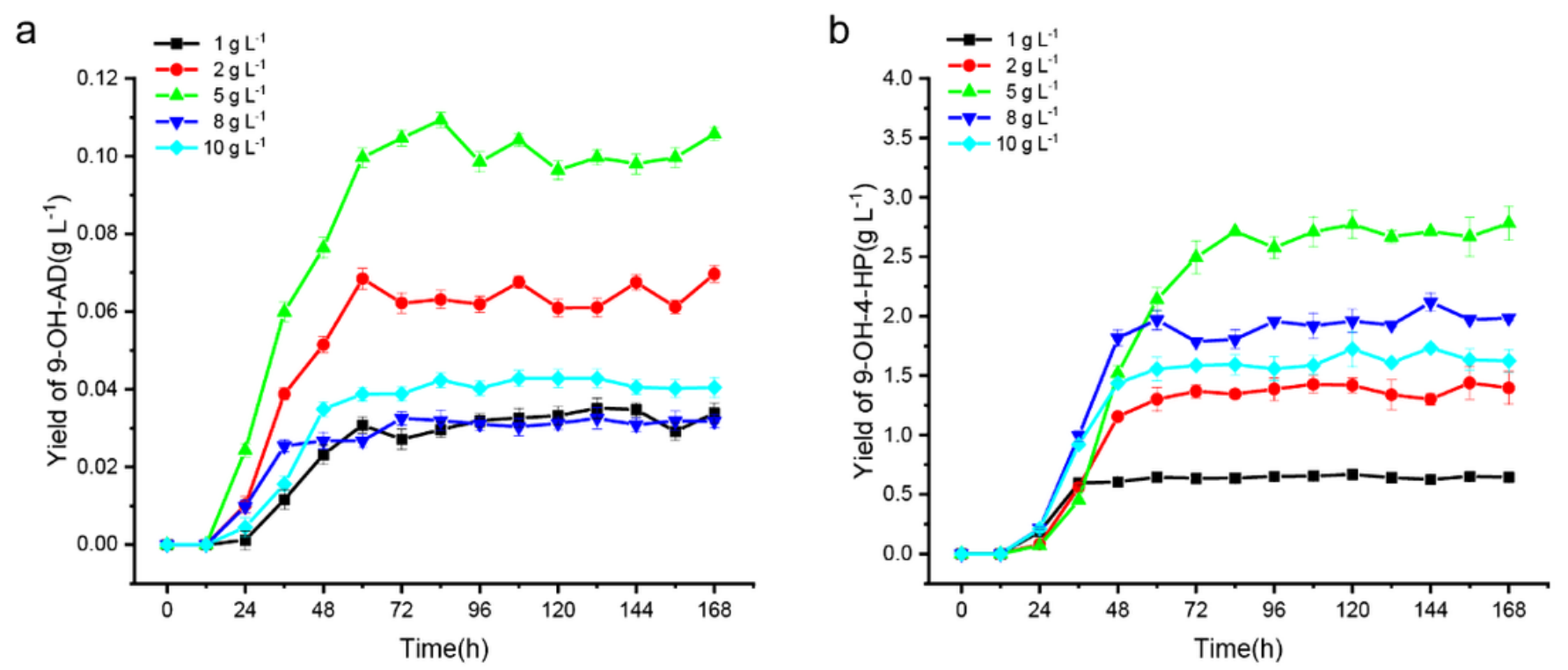

\section{Figure 4}

9-hydroxy steroids accumulation of $\Delta \mathrm{kstD} \Delta \mathrm{hsd} 4 \mathrm{~A} \Delta \mathrm{fadA} 5$ from different concentrations of phytosterols.

(a) Time course of 9-OH-AD accumulation; (b) Time course of 9-OH-4-HP accumulation; As the phytosterols concentration increased, the ability of the strain $\Delta \mathrm{kstD} \Delta \mathrm{hsd} 4 \mathrm{~A} \triangle \mathrm{fadA} 5$ to transform phytosterols was inhibited. 

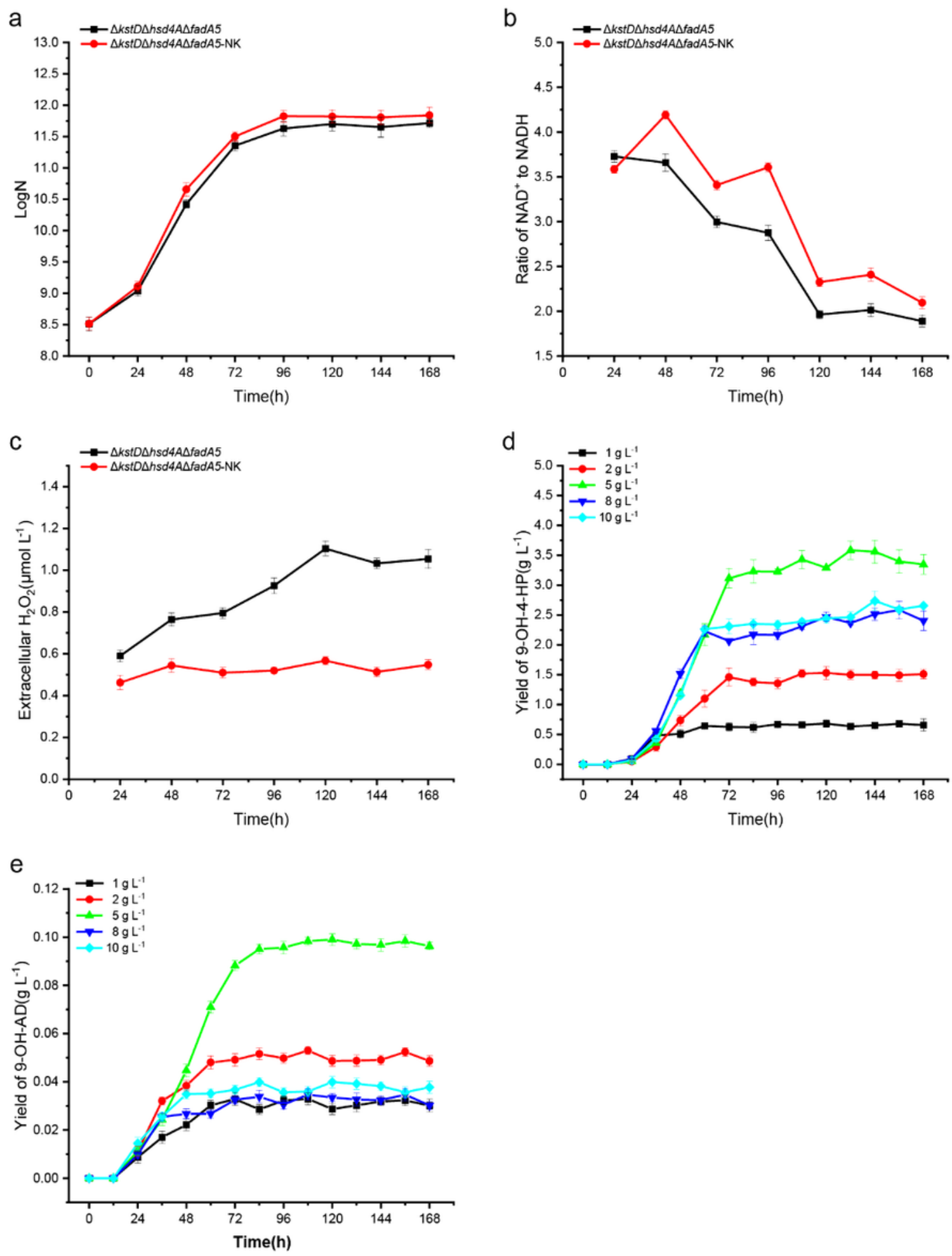

\section{Figure 5}

Time profiles of 9-hydroxy steroids accumulation, extracellular $\mathrm{H} 2 \mathrm{O} 2$ concentration, and the NAD+/NADH ratio of the strain $\Delta \mathrm{kstD} \Delta \mathrm{hsd} 4 \mathrm{~A} \Delta \mathrm{fadA} 5$ and the strain $\Delta \mathrm{kstD} \Delta \mathrm{hsd} 4 \mathrm{~A} \Delta \mathrm{fadA5}-\mathrm{NK}$. (a) the cell growth; (b) intracellular NAD+/NADH ratio; (c) extracellular H2O2 concentration; (d) time course of 9-OH-4-HP accumulation of $\Delta \mathrm{kstD} \Delta \mathrm{hsd} 4 \mathrm{~A} \Delta \mathrm{fadA5}-\mathrm{NK}$ from different concentrations of phytosterols; (d) time course of 9-OH-AD accumulation of $\Delta \mathrm{kstD} \Delta \mathrm{hsd} 4 \mathrm{~A} \Delta$ fadA5-NK from different concentrations of phytosterols. 
Expression of Nox and KatE is beneficial for cell growth, increases the ratio of intracellular NAD+/NADH, decreases the extracellular $\mathrm{H} 2 \mathrm{O} 2$ concentration, and enhance the yield of 9-OH-4-HP under a high concentration of phytosterol.

\section{Supplementary Files}

This is a list of supplementary files associated with this preprint. Click to download.

- AddtionalFiles.docx 